\title{
Genetic control of hepatitis A severity and susceptibility to allergy
}

\author{
Lucienne Chatenoud ${ }^{1,2}$ and Jean-François Bach ${ }^{1,2}$
}

1 Université Paris Descartes, INSERM, Paris, France. ${ }^{2}$ INSERM, Unité 1013, Paris, France.

\begin{abstract}
Hepatitis A virus (HAV) usually results in asymptomatic infection or mild disease, but in a small subset of patients it can lead to severe disease and even liver failure. In this issue of the JCI, Kim et al. identify a polymorphism associated with this increased disease risk in the gene that encodes the HAV receptor-TIM1. Importantly, this TIM1 gene polymorphism protects from the development of atopic diseases in $\mathrm{HAV}$-seropositive individuals.
\end{abstract}

The genetic control of the severity of viral diseases is clearly polygenic. Little is known yet about the nature of the susceptibility genes and about their interaction with environmental factors other than the causal virus. Identifying these genes is, however, of the utmost importance for both fundamental and clinical reasons. This was first attempted by investigating possible associations between infection and major immune response genes such as those encoding class I and class II MHC molecules. One particularly relevant finding was the preferential development of chronic aggressive hepatitis $B$ in hemodialyzed patients with an HLA-A1 B8 haplotype (1). The second method was a candidate gene approach. It has thus been reported that HIV resistance is associated with a partial deletion of CCR5 resulting in a frameshift that generates a nonfunctional receptor (2). It has also been shown that herpes simplex virus-induced meningoencephalitis is associated with mutations in UNC-93B encoding a highly conserved molecule found in the endoplasmic reticulum and involved in Toll-like receptor signaling $(3,4)$. Furthermore, recent reports have revealed an unexpected diversity among the killer cell immunoglobulin-like receptors (KIRs), and the polymorphisms in this gene class and in that of their HLA class I ligands have led to interesting associations in both viral and inflammatory disorders (5).

In this context one must consider with very special interest data reported by Kim et al. in this issue of the JCI (6) demonstrating an association of severe progression of

Conflict of interest: The authors have declared that no conflict of interest exists.

Citation for this article: J Clin Invest. 2011; 121(3):848-850. doi:10.1172/JCI46418. hepatitis A with a particular polymorphism of TIM1, whose encoded protein was identified as the hepatitis A virus (HAV) receptor.

Cloning disease susceptibility genes This work stems from an original observation the authors made in 2001, when, to analyze asthma susceptibility genes located at human chromosome 5q23-q35, they studied congenic mice that differed at the orthologous chromosomal segment (7). With this approach they identified a Mendelian trait encoded by Tapr, the $T$ cell and airway phenotype regulator. Positional cloning identified a gene family that encodes $\mathrm{T}$ cell, immunoglobulin, and mucin domain proteins (TIMs); major sequence variants of this gene family completely cosegregated with Tapr. Interestingly, the human ortholog of TIM-1 is the hepatitis A virus (HAV) receptor, a finding that provided the first robust molecular explanation for the inverse relationship between HAV infection and the development of allergic hypersensitivity (7).

In the present report, the authors identify a polymorphism in TIM1 (157insMTTTVP) that is associated with the development of HAV-induced disease of extreme severity, in contrast to the asymptomatic or mild disease experienced by the majority of patients. The study presented is a case-control, cross-sectional, observational analysis performed on 30 patients presenting with $\mathrm{HAV}$-induced acute liver failure and 102 healthy case controls (blood donors with a past history of mild or asymptomatic HAV infection confirmed by serology).

The first part of the study showed that having one or two copies of the long form of TIM1 - the form that includes the insertion - was associated with a greater risk of developing severe HAV-induced liver failure, while having the short form was associated with protection against severe hepatitis. In addition, the authors performed a series of mechanistic studies to dissect the molecular basis of the modulation of HAVinduced hepatitis severity by TIM1 using several TIM-1-Fc fusion protein constructs containing the IgV domain of TIM-1 and different lengths of the mucin domain in an HAV neutralization assay. They found that the length of the TIM- 1 mucin domain significantly affected binding of HAV; the long form of TIM-1 bound HAV more efficiently than the short form.

\section{Selecting for disease severity?}

As a whole, these results are convincing despite the borderline statistical significance of the human genetics studies, and clearly point to the existence of a population at high risk for HAV disease. The authors suggest that the low frequency of the long form of TIM1 associated with disease severity in the general population might result from selective evolutionary pressure favoring the survival of HAVinfected individuals. Although very interesting, this finalistic hypothesis unfortunately remains pure speculation.

It is particularly notable that the susceptibility gene polymorphism in question is directly associated with a major functional feature of one of the key players in the infection, namely the affinity of the HAV receptor for the virus. This is to our knowledge the first demonstration of such an association that enhances viral disease severity.

\section{Unexpected link with protection from allergic diseases}

An intriguing question is the relationship of severe progression of hepatitis A and HAV-induced protection against allergic diseases, particularly allergic asthma, both associated with the long form of TIM-1. This HAV-associated protection has been known for some time. It was first reported that the frequency of multiple sclerosis and 

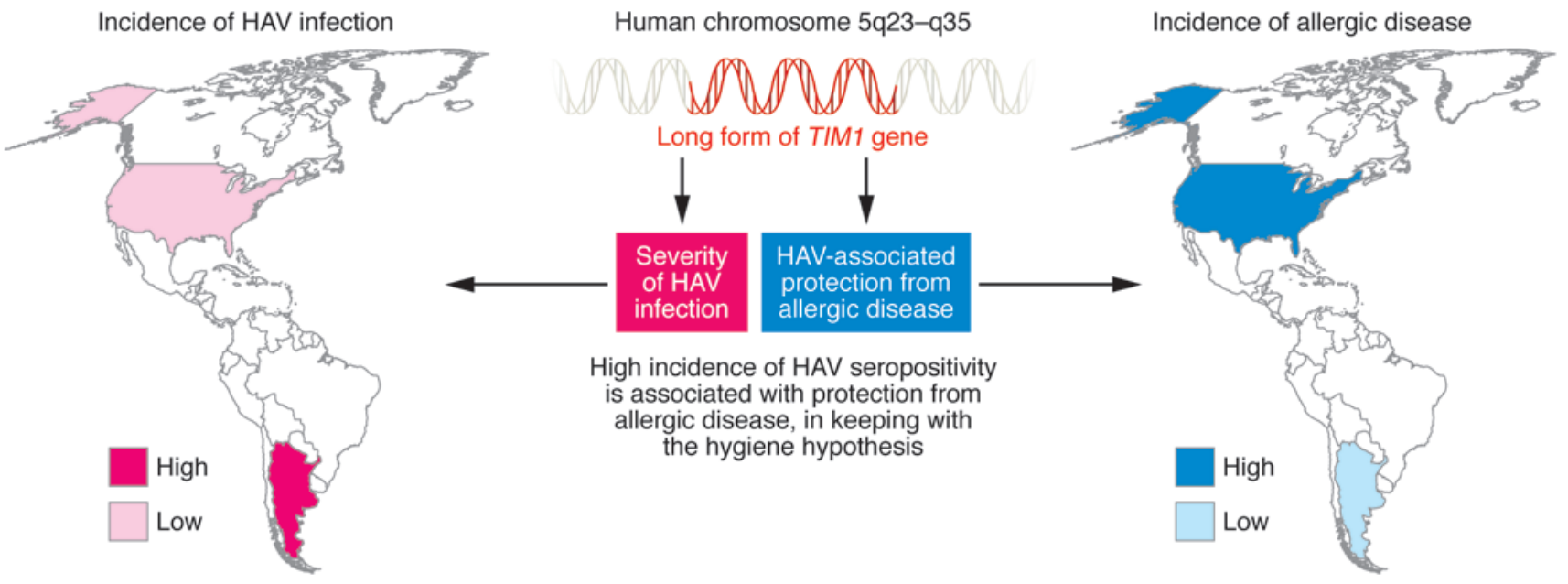

\section{Figure 1}

The immunopathology of hepatitis A and its relationship to allergic disease. There is a reciprocal relationship between the incidence of hepatitis $\mathrm{A}$ and the prevalence of allergic disease, as evidenced by rates of each in the United States and Argentina (where the patients included in the Kim et al. study were recruited). A minority of HAV-infected individuals exhibit a very severe form of the disease, and there is compelling evidence that HAV infection protects from allergic diseases. The work by Kim et al. (6) suggests that both features are under the genetic control of TIM1.

type 1 diabetes, two autoimmune diseases, was negatively correlated with anti-HAV seropositivity, used as a marker of poor hygiene (8). It was thereafter established in a case-control study that patients with anti-HAV antibodies developed reduced frequency of allergic asthma when compared with $\mathrm{HAV}$ antibody-negative subjects (9). Furthermore, it was shown that among anti-HAV antibody carriers, individuals expressing the long form of TIM-1 were protected from allergic asthma (10).

How can one reconcile these observations? An interesting possibility is that the long form of TIM- 1 is associated with a "strong" response to $\mathrm{HAV}$, which will contribute first to severity of hepatitis and secondly to protection against allergy (Figure 1). If this were the case, it would suggest that it is not only the existence of an anti-HAV response that affords protection, but rather its intensity, the underlying molecular mechanisms of which remain to be defined. This explanation would fit with some of the mechanisms proposed to explain the hygiene hypothesis, notably antigenic competition, which suggests that strong anti-infectious immune responses compete with responses against weak antigens such as allergens and autoantigens $(11,12)$.

Returning to the selection pressure of evolution, one may note that according to the hygiene hypothesis, selecting against a virus sensitivity gene such as the 157 insMTTTVP TIM1 polymorphism should lead to a higher frequency of allergic and autoimmune diseases, independent of the global effect of hygiene on contamination by HAV and other infectious agents.

\section{The role of NKT cells}

Kim et al. demonstrate that the expression of the long form of TIM-1 in natural killer T (NKT) cells resulted in greater cytolytic activity against HAV-infected liver cells (6). These data are potentially interesting, yet puzzling. One would like to have the possibility to validate this working hypothesis with more direct evidence in vivo, which is difficult to approach in the human in the absence of pharmacologically safe means to block NKT cell function; this approach is only presently feasible in animal models using mice devoid of NKT cells, such as CD1d-knockout mice.

More importantly, Kim et al. failed to consider the role of other cytotoxic cells, notably NK cells and CD8 ${ }^{+}$cytotoxic lymphocytes, which have well-established roles in hepatitis $\mathrm{B}$ and $\mathrm{C}$ infection (13-15). It is true, as the authors mention, that the only article studying $\mathrm{CD}^{+} \mathrm{T}$ cells in HAV argued against their major role in disease pathophysiology (16), but this was an isolated work that contrasts with the large bulk of evidence accumulated in the study of hepatitis B and C. This is an important point, since it remains formally possible that TIM-1 expression plays a role in forms of cytotoxicity other than that mediated by NKT cells. It is interesting in this regard to mention that $\mathrm{CD}^{+}$cytotoxic $\mathrm{T}$ cells preferentially express various proteins such as PD-1, CTLA4, and TIM-3 in hepatitis $C$ that contribute to their defective function at the origin of disease severity and chronicity (17).

After many years of intensive research, the role of NKT cells in immunopathology still remains elusive. It is tempting to equate the effector function of NKT cells to that of NK cells, with which they share many molecular features, including the expression of activatory and inhibitory KIR receptors, particularly since NK cells are known to play a major role in antiviral defense. Incidentally, it would be important to determine whether NK cells express TIM-1. NKT cells once activated secrete a wide spectrum of cytokines, some of which, such as IFN- $\gamma$, could stimulate conventional cytotoxic T cells. NKT cells have also been shown to control the onset of autoimmune disease, including autoimmune diabetes in NOD mice (18, 19). On the other hand, NKT cells may promote the onset of OVA-induced murine allergic asthma, which emphasizes the complexity of NKT cell function depending on the environment in which they develop (i.e., CD1d-presented ligands and cytokines) (20). This complexity, in addition to the very low percentage of NKT cells in peripheral blood and lymphoid organs in comparison with particular sites, notably the liver, calls into question the importance of NKT cells and their pathophysiological role.

The article by Kim et al. (6) introduces original concepts such as the link between virus receptor affinity and disease severity 
and the shared genetic control of viral disease severity and protection from allergic diseases. In addition, this work reveals a mechanistic link with NKT cells, opening a new avenue for better understanding the pathophysiology of viral diseases in conjunction with more conventional and wellestablished cytotoxic pathways involving $\mathrm{NK}$ cells and antigen-specific CD8 ${ }^{+} \mathrm{T}$ cells.

Address correspondence to: Jean-François Bach, INSERM U1013, Hôpital Necker Enfants Malades, 161 Rue de Sèvres 75015 Paris, France. Phone: 33.1444953.73; Fax: 33.1430623.88; E-mail: jean-francois. bach@academie-sciences.fr.

1. Descamps B, Jungers P, Naret C, Degott C, Zingraff J, Bach JF. HLA-A1, B8-phenotype association and HBs antigenemia evolution in 440 hemodialyzed patients. Digestion. 1977;15(4):271-277.

2. Samson M, et al. Resistance to HIV-1 infection in Caucasian individuals bearing mutant alleles of the CCR-5 chemokine receptor gene. Nature. 1996;382(6593):722-725.

3. Tabeta K, et al. The Unc93b1 mutation 3d dis- rupts exogenous antigen presentation and signaling via Toll-like receptors 3, 7 and 9. Nat Immunol. 2006;7(2):156-164.

4. Casrouge A, et al. Herpes simplex virus encephalitis in human UNC-93B deficiency. Science. 2006;314(5797):308-312.

5. Khakoo SI, Carrington M. KIR and disease: a model system or system of models? Immunol Rev. 2006;214:186-201.

6. Kim HY, et al. A polymorphism in TIM1 is associated with susceptibility to severe hepatitis A virus infection in humans. J Clin Invest. 2011;121(3):1111-1118.

7. McIntire JJ, et al. Identification of Tapr (an airway hyperreactivity regulatory locus) and the linked Tim gene family. Nat Immunol. 2001;2(12):1109-1116.

8. Bach JF. Predictive medicine in autoimmune diseases: from the identification of genetic predisposition and environmental influence to precocious immunotherapy. Clin Immunol Immunopathol. 1994;72(2):156-161.

9. Matricardi PM, Rosmini F, Panetta V, Ferrigno L, Bonini S. Hay fever and asthma in relation to markers of infection in the United States. J Allergy Clin Immunol. 2002;110(3):381-387.

10. McIntire J, et al. Immunology: hepatitis A virus link to atopic disease. Nature. 2003;425(6958):576.

11. Bach JF. The effect of infections on susceptibility to autoimmune and allergic diseases. $N$ Engl J Med. 2002;347(12):911-920.

12. Okada H, Kuhn C, Feillet H, Bach JF. The 'hygiene hypothesis' for autoimmune and allergic diseases: an update. Clin Exp Immunol. 2010;160(1):1-9.
13. Phillips S, Chokshi S, Riva A, Evans A, Williams R, Naoumov NV. CD8(+) T cell control of hepatitis $\mathrm{B}$ virus replication: direct comparison between cytolytic and noncytolytic functions. J Immunol. 2010;184(1):287-295.

14. Satake S, et al. Significant effect of hepatitis C virus specific CTLs on viral clearance in patients with type $\mathrm{C}$ chronic hepatitis treated with antiviral agents. Hepatol Res. 2008;38(5):491-500.

15. Sprengers D, et al. Analysis of intrahepatic HBV-specific cytotoxic T-cells during and after acute HBV infection in humans. J Hepatol. 2006;45(2):182-189.

16. Vallbracht A, et al. Cell-mediated cytotoxicity in hepatitis A virus infection. Hepatology. 1986;6(6):1308-1314.

17. McMahan RH, et al. Tim-3 expression on PD-1+ $\mathrm{HCV}$-specific human CTLs is associated with viral persistence, and its blockade restores hepatocytedirected in vitro cytotoxicity. J Clin Invest. 2010; 120(12):4546-4557.

18. Sharif S, et al. Activation of natural killer T cells by alpha-galactosylceramide treatment prevents the onset and recurrence of autoimmune type 1 diabetes. Nat Med. 2001;7(9):1057-1062.

19. Hong $S$, et al. The natural killer T-cell ligand alphagalactosylceramide prevents autoimmune diabetes in non-obese diabetic mice. Nat Med. 2001; 7(9):1052-1056

20. Lisbonne $\mathrm{M}$, et al. Cutting edge: invariant $\mathrm{V}$ alpha 14 NKT cells are required for allergen-induced airway inflammation and hyperreactivity in an experimental asthma model. JImmunol. 2003;171(4):1637-1641. 